\title{
Improving emergency department flow through Rapid Medical Evaluation unit
}

Lucas Chartier, Timothy Josephson, Kathy Bates, Meredith Kuipers

University Health Network, Canada

\begin{abstract}
The Toronto Western Hospital is an academic hospital in Toronto, Canada, with an annual Emergency Department (ED) volume of 64,000 patients. Despite increases in patient volumes of almost six percent per annum over the last decade, there have been no commensurate increases in resources, infrastructure, and staffing. This has led to substantial increase in patient wait times, most specifically for those patients with lower acuity presentations. Despite requiring only minimal care, these patients contribute disproportionately to ED congestion, which can adversely impact resource utilization and quality of care for all patients.
\end{abstract}

We undertook a retrospective evaluation of a quality improvement initiative aimed at improving wait times experienced by patients with lower acuity presentations. A rapid improvement event was organized by frontline workers to rapidly overhaul processes of care, leading to the creation of the Rapid Medical Evaluation (RME) unit - a new pathway of care for patients with lower acuity presentations. The RME unit was designed by re-purposing existing resources and re-assigning one physician and one nurse towards the specific care of these patients. We evaluated the performance of the RME unit through measurement of physician initial assessment (PIA) times and total length of stay (LOS) times for multiple groups of patients assigned to various ED care pathways, during three periods lasting three months each.

Weekly measurements of mean and 90th percentile of PIA and LOS times showed special cause variation in all targeted patient groups. Of note, the patients seen in the RME unit saw their median PIA and LOS times decrease from 98min to $70 \mathrm{~min}$ and from $165 \mathrm{~min}$ to $130 \mathrm{~min}$, respectively, from baseline. Despite ever-growing numbers of patient visits, wait times for all patients with lower acuity presentations remained low, and wait times of patients with higher acuity presentations assigned to other ED care pathways were not adversely affected.

By specifically re-purposing a fraction of existing staff, resources, and infrastructure for patients with lower acuity presentations, we were able to streamline their care and decrease wait times in the ED. These results were achieved through the incremental improvements afforded by rapidly cycling through PDSA cycles, with strong frontline staff involvement and continuously eliciting feedback for improvement. We believe the model to be replicable in other academic medical centres.

\section{Problem}

The Toronto Western Hospital (TWH) is an urban tertiary academic medical center in Toronto, Canada, with an annual Emergency Department (ED) volume of more than 64,000 patients. Two thirds of the patients presenting to the TWH ED are assigned to the Fast Track (FT) area, designed and designated for rapid assessment and medical treatment of patients with more minor clinical concerns. The remaining sicker patients are triaged to the acute or subacute areas.

Over the last decade at the TWH, there has been an average increase in ED patient volumes of almost $6 \%$ per annum, with low acuity patients representing the largest and fastest growing subgroup. Without any major structural investments or improvements during the same period, however, spatial constraints and human resources limitations have created significant pressures to assess and treat these additional patients in a timely manner. This has resulted in disproportionately longer wait times experienced by the lowest acuity cohort of patients.

\section{Background}

When patients present to the ED, the first step in their care usually includes a nursing triage assessment, followed by the assignment of an acuity score to indicate the severity of their current medical condition. This process is known as triage, and it results in the prioritization of the subsequent assessment and management of patients according to their acuity score.

Canadian EDs use the Canadian Triage and Acuity Scale (CTAS) to triage patients based on presenting complaint, vital signs and select modifying factors [1]. A CTAS score ranks a patient from one to five, ranging from emergent conditions requiring immediate nursing and physician attention (CTAS-1 category), to non-urgent conditions, with a recommended initial physician assessment time of two hours maximum (CTAS- 5 category). Compared to sicker patients, those with lower acuity scores are often sent to a physically distinct area of the ED that is better suited to serve their individual needs. In the TWH ED, this area is called the Fast Track (FT) area. Patients who meet the criteria for receiving care in the FT area can likely be assessed, treated, and discharged within four hours (this target time represents an institutional target, and is not 
legislatively mandated).

Once sent to different sections of the ED, patients are then seen by the ED physician in a decreasing order of acuity, based on their CTAS score. At the TWH ED, two thirds of patients are triaged to the FT area, and most of these FT patients have a CTAS score of three, four, or five. In this context, patients designated as CTAS-3 may occasionally jump the queue in front of CTAS- $4 / 5$ patients, resulting in extended length of stay for these lower acuity patients. Thus, the least sick patients tend to contribute disproportionately more to the congestion of the ED, a bottleneck that has been associated with worse outcomes for both admitted and discharged patients [2,3]. The resultant increased burden on ED staff and resources can lead to decreased morale among staff, worse care for all patients, and fewer resources and staff available per patient $[4,5]$.

\section{Baseline measurement}

Although the quality improvement (QI) project described in this manuscript was prospectively developed, implemented, and measured, the full evaluation of its impact on wait times was only retrospectively achieved. As a result, some of the metrics were determined post hoc.

The time-based metrics selected for this study are both institutional benchmarks selected for quality of care and mandatory reporting measures to governmental health agencies, and were abstracted from an institutional database. The first measure is the time to Physician Initial Assessment (PIA time), which is the length of time experienced by patients between triage and the initial assessment performed by a physician or his/her delegate (i.e. trainee, physician assistant, or nurse practitioner). The second measure is total of Length of Stay (LOS) time, which is the length of time experienced by patients between triage and discharge from the ED (whether to an inpatient unit or out of hospital). We only included data of patients for whom both PIA and LOS times were documented. In this paper, the two measures together are referred to as "wait times". We elected to measure both the mean and the 90th percentile of wait times because they focus on two separate but important metrics. Although means are affected by the disproportionately long wait times of a minority of patients, they provide an excellent barometer of the 'average' service provided to our patients and the wait times they can expect. The 90th percentile, on the other hand, help understand the maximum wait times experienced by nine patients out of ten patients, and are not affected as much by outliers. As such, they help us understand where issues may arise that affect many of our patients and that should be analyzed further.

The outcome measures selected are the PIA and LOS times for patients with a CTAS score of four or five (reported together as CTAS-4/5), which was the target population of this QI project. The balancing measures are the PIA and LOS times for higher acuity (sicker) patients with a CTAS score of one to three (reported together as CTAS-1/2/3). As a process measure, we also collected ED patient volumes, as increased volumes typically correlate with increased wait times.
Historical registration data indicate that ED patient volumes are highly cyclical based on time of year, day of week, and even hour of day. Such predictable periodicity allows for planning and optimization of staffing and resources during times of high and low ED traffic. Given this knowledge of ED traffic and the planned length of time of the implementation and evaluation of our intervention (three months), we elected to obtain two baseline measurements: the three months immediately preceding our intervention (termed 3-mo PRE), and a three-month period exactly one-year prior (termed 1-yr PRE; Figure 1). These two baselines allowed us to account for any seasonal variation that would normally be expected between winter (our 3-mo PRE measurement spanned December to February) and spring (the intervention was implemented and evaluated from March to May). Historical registration data also indicated that ED volumes were highest between the hours of 11:00 and 19:00 daily. This trend informed both the baseline measurement time periods and the design of the intervention.

Independent samples t-tests were conducted to compare mean wait times between the different time points for the two different patient groups. Statistical analysis was performed using Microsoft Excel and $p$ values $<0.05$ (2-sided) were considered significant.

As predicted, our baseline measurements indicated seasonal variation in ED volumes for all patient groups, with higher volumes in the 1-year PRE period. This is consistent with patterns seen in previous years, and unrelated to any other quality improvement initiatives or factors inherent to our ED. Of note, there were reductions in all the measured variables of wait times for CTAS- $4 / 5$ patients between the 1-year PRE and the 3-mo PRE baselines (Table 1). We attribute some of the reduction in wait times over this period to the difference in patient volumes.

See supplementary file: ds6339.pdf - "Figure 1 and Table 1"

\section{Design}

Given the relatively long wait times experienced by the CTAS- $4 / 5$ patients in our ED, we elected to focus on this particular patient population. We hypothesized that separating the lowest acuity patients from the larger group of patients seen in the FT area would improve the timeliness of their care. The initial conceptualization and implementation of this project was undergone as part of a multidisciplinary four-day LEAN event. This rapid improvement event engaged front-line workers to drastically and quickly alter the current processes of care. The result was the creation of the Rapid Medical Unit (RME) unit, which was implemented in March 2014.

The RME unit was used to assess and treat those 'very low' acuity CTAS-4/5 patients. Specifically, it targeted patients who were stable and ambulatory, with non-complex prior medical histories and current needs, with minimal expected investigations and interventions, and who were not expected to need a specialist consultation. Pre-determined guidelines enabled the triage nurse to designate a patient for the RME unit at the onset of their ED visit, separating them from the other FT patients. No increase in staffing or resources was planned for operation of the RME; as such the up- 
front costs of the RME were negligible. The RME unit occupied a previously inefficiently utilized semi-enclosed area of the ED, physically distinct from the FT area, and corresponding roughly to the size of one large treatment room. The room was outfitted with one small assessment bed, as well as three chairs for patients to sit and wait for their treatment or the results of their investigations. No new equipment or supplies were purchased; rather materials were repurposed from unused locations within the hospital. One physician (MD) and one registered nurse (RN) who were already scheduled were designated to staff the RME unit. No other MD or $\mathrm{RN}$ clinical responsibilities were affected elsewhere in the ED.

\section{Strategy}

All Plan-Do-Study-Act (PDSA) cycles were tested and implemented during the first three months after the implementation of the RME unit.

PDSA Cycle 1:

During the first PDSA cycle, the RME unit functioned as aforementioned for eight hours every day. The MD and RN shifts were left unchanged from their original scheduled times, with the RME unit starting partway through the 12-hour RN shift. As a result, the designated $\mathrm{RN}$ was required to change his/her patient assignment in the middle of the shift, requiring handover of patients and causing delays. Moreover, union-mandated nursing breaks were often scheduled to occur early on in the MD shift, leading to lack of adequate support. An RN from a different area of the ED would often fill in and perform the MD orders, often causing a disruption in the flow of the ED as a whole. It was perceived that these elements contributed significantly to a loss of efficiency in the RME unit. As a result, the eight-hour MD shift was moved to an earlier start time to permit a full and concurrent nursing assignment in the RME unit, without compromising nursing coverage elsewhere in the ED.

PDSA cycle 2:

Junior physicians in training (i.e. medical residents, or registrars) initially were scheduled to work with attending staff physicians (i.e. consultants) in the RME unit, as per the normal scheduling practices in a teaching hospital. It quickly became evident, however, that the nature of the single-bed, expedited assessments intended by the RME unit precluded quality bedside teaching. As a result, it was decided that the RME shift would be tested as a non-teaching shift to increase efficiency, and provide better educational opportunities to the trainees through scheduling on other assignments.

PDSA cycle 3:

The initial guidelines for identifying patients suitable to the RME unit were left intentionally vague, subject to interpretation by the triage $\mathrm{RN}$ within the whole clinical spectrum. The general specifications were well-appearing, non-critical, ambulatory patients who would likely require few and simple investigations (e.g., plain x-rays or basic blood work). There was a learning curve from all members of the team with regards to the selection of appropriate patients, and continuous feedback from the MD-RN teams was elicited and considered. Over time, the triage RNs - who on other days may be scheduled in the RME unit - became much more comfortable and proficient at selecting the most appropriate patients for the RME unit.

PDSA cycles 4 and beyond:

Multiple additional PDSA cycles were performed to constantly improve the RME experience, both for the patients and the providers caring for them. Feedback was collected on a daily basis, analyzed weekly, and change ideas implemented continuously. The workstation and flow processes underwent weekly tests of change, most of which were implemented when shown to be successful. These small incremental changes, although relatively simple in their individual effects, collectively boosted both the efficiency and the morale of the clinicians working in the RME unit. Examples of changes included improved lighting to perform minor procedures, updated stocking of commonly-used equipment such as gloves, suture trays and material, and additional computer monitors and information technology access.

\section{Results}

We focused our assessment on the immediate effects of the presence of the RME unit during the first three months postimplementation, when the aforementioned PDSA cycles were tested. Table 2 shows the mean and P90 of the PIA and LOS times for the post-implementation period and their difference with baseline measures. Despite no additional resource utilization, the wait times for all CTAS- $4 / 5$ patients decreased significantly after the implementation of the RME. Patients assessed in the RME experienced the shortest wait times of all patients: on average, RME patients waited 12 minutes less to see a physician and stayed in the ED 36 fewer minutes overall compared to other CTAS-4/5 patients seen elsewhere in the department (see Table 2).

As shown in Table 3, our balancing measure of PIA and LOS times for CTAS-1/2/3 patients seen at concurrent times changed modestly over the measurement time period. Compared to 3-mo PRE, all PIA and LOS variables in the POST time period increased, while only P90 LOS increased compared to 1-yr PRE. These POST changes occurred alongside commensurate growth in patient volumes by $11 \%$ and $15 \%$ compared to 3-mo PRE and 1-yr PRE, respectively. Overall, there was a fairly narrow range in wait time change for CTAS- $1 / 2 / 3$ patients (from -9 minutes to +16 minutes), few of which were statistically significant in either direction.

To visualize the weekly changes in PIA and LOS, the mean and 90th percentile (P90) values of each outcome measure were plotted in separate statistical process control (SPC) charts (Figures 2 to 5). As would be expected, the SPC charts pertaining to the PIA (Figures 2 and 3 ) show reduced variability compared to the ones pertaining to the LOS (Figures 4 and 5), and those pertaining to the means (Figures 2 and 4 ) less than the P90 ones (Figures 3 and 5). All charts point to different issues that may require different solutions: mean times refer to all patients whereas P90 times 
exclude those at the extreme of wait times. PIA times refer to the upfront work necessary to get patients seen by physicians, whereas LOS times also include all those processes pertaining to the diagnosis and management of patients. For each of the four outcome measures, the post-implementation RME data (represented by the green lines in each figure) demonstrated special cause variation, as indicated by a run of at least eight consecutive data points falling below the mean of the sample.

See supplementary file: ds6566.pdf - "Tables 2,3; Figures 2-5"

\section{Lessons and limitations}

The RME unit was a resounding success for our ED, despite growing patient volumes. Our target population - the CTAS-4/5 patients - saw decreased wait times at the 90th percentile by up to 78 minutes compared to patients following the usual FT care pathway prior to implementation of the RME. There were modest impacts on the wait times of sicker patients, few of which were statistically significant. The relative volume of patients seen in each area of the ED remained the same, such that workflow of other clinicians in the ED was unchanged.

Many small tests of change were conducted during the development of the RME through the four-day rapid improvement event. The scale of measurement that was used to evaluate change in wait times - weekly mean and ninetieth percentile - was not sufficiently sensitive to capture changes on a daily basis. Capturing the selected outcomes for analysis on a daily scale was unrealistic due to the inherent fluctuations in day-to-day patient volumes.

Following the three-month RME implementation period, other improvement projects occurred and were ongoing. The project leads felt that the data would be too contaminated to detect sustainability specific to the RME, and therefore the costs of data abstraction were not justified. However, the overall wait times for CTAS-4/5 patients have remained low over time despite an evergrowing number of visits. The gains achieved through the RME implementation required limited resources, which is why the RME unit continues to be a mainstay in our ED to this day, with very limited changes since the initial implementation.

While the wait times for the overall group of CTAS- $4 / 5$ patients decreased, some patients seen in the FT area did experience slightly lengthier wait times (denoted by the dashed blue line in Figures 2-5). This small increase was likely in part due to the redirection of lowest acuity patients to the RME zone, leaving a relatively larger number of more complex patients in the FT who are expected to have a longer length of stay. It should be noted that the increased wait times for CTAS-4/5 patients seen in FT was not significant despite increasing patient volumes across the whole department during the three-month post-implementation period relative to both baseline measurements.

One limitation of this project, inherent to most quality improvement (QI) initiatives, was the dynamic nature of the ED during implementation of the RME unit. Other improvement projects occurred concurrently with this QI initiative, such that isolated evaluation of the effect of the RME unit was difficult. Although individually smaller in scope and impact than the RME unit, the combination of these changes may have contributed to the improvement in the patient wait times for FT patients. However, these changes were unlikely to have directly affected the wait times of RME patients, and thus have little impact on our outcome measures.

Implementing a new model of care in our ED presented several unique challenges. As a department, we climbed a steep learning curve in learning to facilitate the adjustment of staff to a completely different workflow. As opposed to the usual process whereby physicians work alongside a few nurses to treat many patients in different areas of the ED, the RME model had one physician and one nurse simultaneously assessing the same patient at any given time. Given the large size of our group of clinicians (69 physicians and 110 ED nurses), MDs and RNs did not rotate through the RME regularly. This may have decreased the gains in efficiency due to knowledge retention and work strategy adaptation.

Finally, although improvement in the wait times of PIA and LOS may have led to higher patient satisfaction, as previously documented in the literature, we did not directly capture the patient experience or other patient-centred outcomes [6]. In order to influence our next improvement cycles, it may be useful and interesting to determine which part of the patient stay is ranked highest and lowest in our ED.

\section{Conclusion}

The RME unit was a solution created to mitigate the problem of lengthy delays in the assessment of lower acuity patients in our urban hospital. By specifically designating a fraction of existing staff, resources, and infrastructure for these patients, we were able to streamline their care and decrease the length of time they spent waiting in the ED. The mean physician initial assessment and the length of stay times were significantly reduced over the course of the three months after the implementation of the intervention. Most notably, special cause variation was noted for each of the four outcome measures analyzed. These results were achieved through the incremental improvements afforded through multiple PDSA cycles. In hindsight, formal feedback on the patient and staff experience would have provided an interesting qualitative perspective on the process and outcome of this project.

\section{References}

1 Bullard MJ, Chan T, Brayman C, et al. Revisions to the Canadian Emergency Department Triage and Acuity Scale (CTAS) Guidelines. CJEM. 2014;16(6):485-9

2 Sun BC, Hsia RY, Weiss RE, et al. Effect of emergency department crowding on outcomes of admitted patients. Ann Emerg Med. 2013;61:605-611.

3 Guttmann A, Schull MJ, Vermeulen MJ, et al. Association between waiting times and short term mortality and hospital 


\section{BMJ Quality Improvement Reports}

admission after departure from emergency department: population based cohort study from Ontario, Canada. BMJ. 2011;342:d2983

4 Rondeau KV, Francescutti LH. Emergency department overcrowding: the impact of resource scarcity on physician job satisfaction. J Healthc Manag. 2005;50(5):327-40

5 Pines JM, Isserman JA, Kelly JJ. Perceptions of Emergency Department Crowding in the Commonwealth of Pennsylvania. West J Emerg Med. 2013;14(1):1-10

6 Preyde M, Crawford K, Mullins L. Patients' satisfaction and wait times at Guelph General Hospital Emergency Department before and after implementation of a process improvement project. CJEM. 2012;14(3):157-168

\section{Declaration of interests}

Nothing to declare.

\section{Acknowledgements}

The authors wish to recognize the significant contribution of the late Jill Smirnis, TWH ED Nurse Manager, and Jason Dickson, TWH ED Administrative Assistant, for their leadership during the rapid improvement event that led to the creation of the RME unit, as well as the entire TWH ED staff who continue to make the RME unit a success every day.

\section{Ethical approval}

According to the policy activities that constitute research at the University Health Network (UHN), this work met criteria for operational improvement activities exempt from ethics review. UHN assesses projects for ethical review based on the nature of inquiry and the level of risk posed to participants, facilitated by completion of the ARECCI Ethics Screening Tool. This project was deemed a quality improvement initiative with minimal risk (ARECCI score $=0$ ) 